\title{
Uma interpretação estatística do PIB, da PNAD e do salário mínimo
}

\author{
EDMAR BACHA \\ RODOLFO HOFFMANN*
}

RESUMO: Por que a renda familiar na Pesquisa Nacional de Domicílios (PNAD) cresceu muito mais rápido do que o consumo das famílias no PIB brasileiro de 2011 a 2012? Para responder essa pergunta, começamos a partir de uma visualização da importância do salário mínimo na PNAD na distribuição de renda, e de uma hipótese de que a subestimação da renda nos levantamentos da PNAD está concentrada naquelas famílias cujos rendimentos não acompanham o salário mínimo . Elaboramos uma equação para explicar a diferença entre o crescimento do rendimento agregado familiar na PNAD e o crescimento do consumo das famílias nas contas nacionais em função da mudança no salário mínimo. As estimativas empíricas dessa equação sugerem que o comportamento do salário mínimo tem sido um componente importante na explicação das diferenças de crescimento da renda entre a PNAD e as contas do PIB.

PALVRAS-CHAVE: Salário mínimo; contas nacionais; estimativa de renda.

ABSTRACT: Statistical interpretation of the GDP, the PNAD and the minimum wage. Why has household income in the National Household Survey (PNAD) grown so much faster than household consumption in Brazil's GDP from 2011 to 2012? To provide an answer to this question, we start from a visualization of the importance of the minimum wage in PNAD's income distribution and from a hypothesis that the underestimation of incomes in the PNAD surveys is concentrated in those families whose incomes do not follow the minimum wage. We then derive an equation to explain the difference between household income growth in the PNAD and household consumption growth in the national accounts as a function of the change in the minimum wage. Our empirical estimates of this equation suggest that the behavior of the minimum wage has been an important component in the explanation of differences in income growth between the PNAD and the GDP accounts. KEYWORDS: Minimum wage; national accounts; income survey. JEL Classification: D31.

\footnotetext{
* Diretor do Instituto de Estudos de Política Econômica/Casa das Garças; e-mail: edmarbacha@gmail. com; Professor Senior da Escola de Agricultura Luiz de Queiróz da Universidade de São Paulo - ESALQUSP, e-mail: hoffmannr@usp.br. Submetido: 26/Dezembro/2013; Aprovado: 22/Agosto/2014.
} 


\section{INTRODUÇÃO}

A renda domiciliar real per capita da Pesquisa Nacional por Amostra de Domicílios (PNAD) cresceu 7,9 por cento entre 2011 e 2012, enquanto que o PIB real per capita cresceu apenas 0,2 por cento. Essa enorme discrepância reacendeu o debate sobre qual das duas medidas reflete melhor a evolução da economia do país, o pibinho ou a pnadona ${ }^{1}$ ?

Parte da discrepância poderia, em tese, ser explicada por uma diferença de deflatores. A renda real da PNAD é calculada deflacionando-se os valores nominais pelo INPC, ou seja, pelos preços médios de uma cesta de consumo das famílias que inclui os bens importados. Já o PIB real envolve o cálculo do deflator implícito do PIB, que reflete os preços médios da cesta de bens e serviços produzidos pelo país, a qual inclui os bens exportados. Dessa forma o INPC incorpora os preços das importações, enquanto o deflator implícito do PIB incorpora os preços das exportações. Quando as relações de troca do país melhoram (ou seja, quando a relação entre os preços das exportações e os preços das importações aumenta), como ocorreu de 2004 a 2011, o deflator implícito tende a superar o INPC e isso explica boa parte da diferença entre o crescimento da renda real da PNAD e o do PIB real no período. Mas esse fator não teve relevo nas variações observadas entre 2011 e 2012, já que o deflator implícito cresceu 5,4 por cento, enquanto o INPC, considerando o período de referência da PNAD, cresceu mais do que isso, ou 5,8 por cento.

Outra razão para a discrepância é que a PNAD mede apenas a renda das famílias, que é um conceito mais restrito do que o do PIB (que incorpora a renda das empresas e do governo, além de medir melhor a renda das famílias do que a PNAD o faz). Isso pode ser remediado usando-se, no lugar do PIB, apenas um de seus componentes, a saber, o consumo das famílias das contas nacionais. As variações percentuais do consumo das famílias nas contas nacionais deveriam ser bem próximas das variações da renda das famílias na PNAD (desde que o grau de subestimação das rendas pela PNAD permaneça aproximadamente constante), inclusive porque os deflatores relevantes para as duas séries devem ter comportamento semelhante (respectivamente o deflator implícito do consumo das famílias nas contas nacionais e o INPC). O problema de as rendas da PNAD referirem-se apenas a setembro e o consumo das famílias estar disponível apenas para cada trimestre pode ser minimizado estimando-se o consumo das famílias de setembro através de uma média ponderada dos valores dessa variável para o terceiro e o quarto trimestre de cada ano.

Quando se adotam essas correções, o que se constata é que, entre setembro de 2011 e setembro de 2012, o consumo real per capita das famílias nas contas nacionais cresceu apenas 2,7 por cento - em comparação com os 7,9 por cento de

\footnotetext{
${ }^{1}$ A extraordinária diferença entre o crescimento do PIB e o crescimento da renda declarada na PNAD de 2011 a 2012 foi ressaltada nos três primeiros parágrafos de documento do IPEA (2013). O tema foi analisado com mais profundidade em Baumgarten de Bolle e Simões (2013). Ver, também, Bacha (2013).
} 
crescimento da renda real domiciliar per capita na PNAD. Ou seja, persiste uma enorme diferença entre as contas nacionais e a PNAD.

Neste artigo, exploramos a hipótese de que essa diferença entre as contas nacionais e a PNAD se deva ao comportamento do salário mínimo. Especificamente, nossa hipótese é que, quanto maior for a variação do salário mínimo, maior será a diferença entre as variações da renda da PNAD e as do consumo das famílias nas contas nacionais. Para chegar a essa hipótese, partimos da constatação que, em nível agregado, a renda das famílias declarada à PNAD é cerca de 3/4 do valor do consumo das famílias nas contas nacionais no mesmo ano. Pressupomos então que essa subestimativa está associada a dois fenômenos. O primeiro é que a proporção das famílias pesquisadas pela PNAD cuja renda segue o salário mínimo deve ser maior do que a embutida no cálculo do consumo das famílias nas contas nacionais. Isso por causa da maior dificuldade, na PNAD, de obter informações corretas sobre a renda das famílias no setor informal e das famílias com renda mais elevada. A segunda razão é que a subestimativa da renda declarada à PNAD pelas famílias cuja renda segue o salário mínimo deve ser menor do que a das famílias cuja renda não segue o salário mínimo. Isso porque quando a renda segue o mínimo é mais fácil a identificação do crescimento efetivo dessa renda.

O que constatamos nos dados é que, quando se leva em conta a variação do mínimo, reduz-se significativamente a diferença não explicada entre as variações do consumo das famílias nas contas nacionais e as da renda das famílias na PNAD.

O restante desse texto está organizado da seguinte forma. Na próxima seção são analisados dados da PNAD com o objetivo de mostrar como o valor do salário mínimo afeta a distribuição da renda revelada por essa pesquisa do IBGE. Na seção seguinte elaboramos um modelo simples a partir das ideias expostas no parágrafo anterior para derivar uma equação para explicar a diferença entre as variações da renda da PNAD e as do consumo das famílias nas contas nacionais. A estimação dessa equação é feita na quarta seção, utilizando dados anuais para o período de 1996 a 2012. A quinta seção conclui.

\section{A IMPORTÂNCIA DO SALÁRIO MÍNIMO NAS RENDAS DECLARADAS NA PNAD}

Para mostrar a importância do salário mínimo na determinação da forma da distribuição da renda no Brasil, segundo a PNAD, é melhor considerar as rendas individuais, e não a renda domiciliar per capita (RDPC), pois nessa um rendimento individual igual ao salário mínimo já está dividido pelo número de membros do domicílio. Uma alternativa usual é considerar apenas o rendimento das pessoas ocupadas. Mas então deixamos de captar apropriadamente a influência do grande número de aposentadorias e pensões iguais ao salário mínimo. Optamos, então, por analisar a distribuição da renda entre todas as pessoas de 10 anos ou mais de idade com algum rendimento de qualquer tipo (rendimento de todas as fontes).

Em cada ano foi definido um intervalo de renda em torno de um salário mínimo. 
Sendo $S$ o valor do salário mínimo, foram considerados aproximadamente iguais ao salário mínimo (pertencentes à categoria SM1) os valores do rendimento pessoal de todas as fontes que ficassem entre o valor arredondado de $S / 1,095$ e o valor arredondado de 1,095 $\times$ S. Em 2012, por exemplo, os limites são 622/1,095 = 568 e 1,095 $\times 622=681$ e assim foram considerados como pertencentes à categoria SM1 os rendimentos pessoais de todas as fontes maiores do que 568 e menores do que 681 .

O Gráfico 1 mostra a evolução, de 1995 a 2012, da proporção de pessoas de 10 anos ou mais de idade com algum rendimento que foi classificada na categoria SM1. Mostra, também, a evolução da correspondente participação na renda total declarada por essas pessoas no Brasil (exclusive a área rural da antiga região Norte). Enquanto a proporção de pessoas com rendimento próximo ao salário mínimo cresce de 17 por cento para 22 por cento, a participação da sua renda no total cresce de $4 \%$ para quase $10 \%$.

Gráfico 1: O crescimento da porcentagem de pessoas com 10 anos ou mais de idade com rendimento próximo ao salário mínimo e da correspondente porcentagem do rendimento total

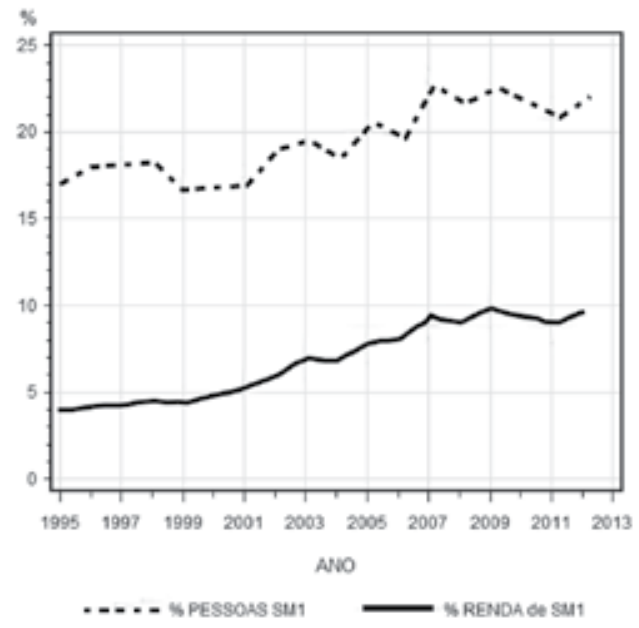

Comparamos, a seguir, as distribuições do logaritmo desse rendimento em 1995 e 2012. Para isso as rendas de 1995 são inflacionadas e os dados da área rural da antiga região Norte são excluídos da amostra de 2012. A função da densidade em cada ano é estimada pelo método "kernel", que considera uma "janela" que se desloca ao longo da distribuição ${ }^{2}$. Foram considerados dois valores para a largura dessa janela. Uma janela mais estreita capta variações localizadas na forma da distribuição, como, por exemplo, a tendência de as pessoas declararem valores redondos. O uso de uma janela mais larga, por outro lado, suaviza a função da densidade e ressalta a forma geral da distribuição.

\footnotetext{
${ }^{2}$ Soares (2004) utilizou esse método para mostrar a influência do salário mínimo na forma da distribuição do rendimento das pessoas ocupadas. Ver, também, Hoffmann (2008).
} 
As curvas estimadas nos dois anos estão no Gráfico 2. A linha tracejada é obtida usando uma janela mais larga e a linha contínua usando uma janela mais estreita. Linhas verticais assinalam as posições correspondentes ao salário mínimo e a valores redondos das rendas nominais, em centenas de reais.

Note-se, na linha contínua para 2012, o pico no valor do salário mínimo (R\$ $622,00)$ e os picos secundários nas posições correspondentes a $\mathrm{R} \$ 1.200,00$ e R \$ $2.000,00$.

$\mathrm{Na}$ linha contínua para 1995 o pico mais elevado também ocorre no valor do salário mínimo, cujo valor nominal era $\mathrm{R} \$ 100,00$, que corresponde a 302,47 reais de setembro-outubro de 2012.

Gráfico 2: Estimativas das funções de densidade da distribuição da renda entre pessoas de 10 anos ou mais de idade com algum rendimento, no Brasil, em 1995 e 2012.

1995

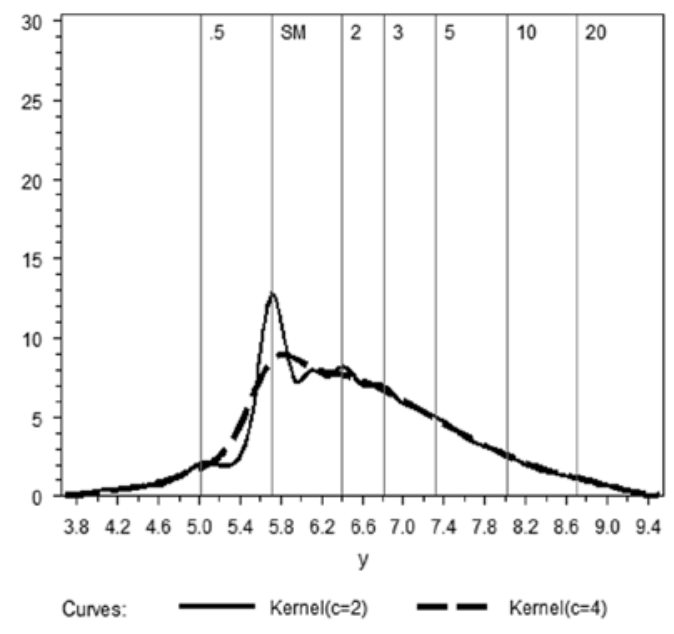

2012

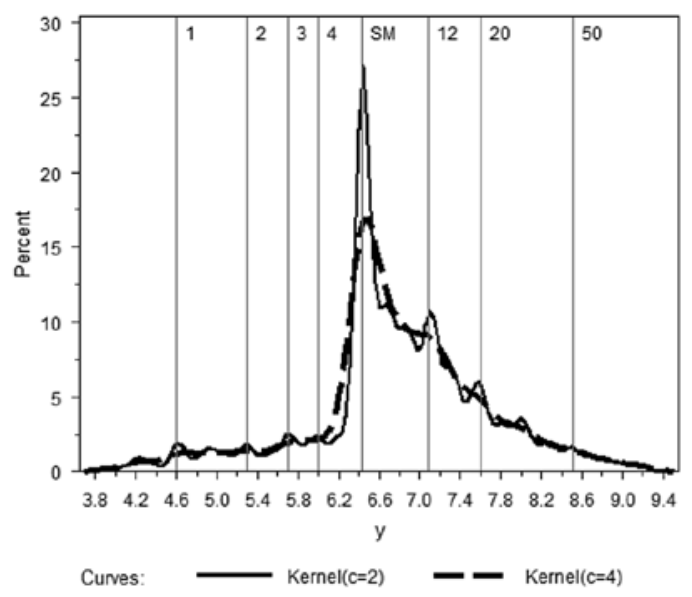


Observa-se que há, entre 1995 e 2012, um deslocamento da distribuição para a direita, associado ao crescimento da renda média real. Mas é impressionante a mudança da forma da distribuição associada ao crescimento de 106 por cento no salário mínimo real. É como se a massa de rendimentos fosse "empurrada e espremida" pelo lado esquerdo.

\section{UM MODELO SIMPLES}

Propomo-nos a derivar uma expressão ligando a evolução do salário mínimo à diferença observada entre o crescimento da renda domiciliar per capita da PNAD e o crescimento do consumo per capita das famílias nas contas nacionais.

Supomos, para isso, haver apenas dois tipos de famílias: aquelas cuja renda per capita segue o salário mínimo e aquelas cuja renda per capita não segue o salário mínimo. Por hipótese o consumo dos dois tipos de família é sempre igual a sua renda. Na PNAD, as famílias cuja renda segue o salário mínimo representam uma proporção $a$ do total das famílias e as segundas, a proporção $(1-a)$. Nas contas nacionais, as primeiras constituem uma proporção $b$ do total das famílias e as segundas, a proporção $(1-b)$. Supomos que $a>b$, ou seja, há mais famílias cuja renda (leia-se: consumo) segue o salário mínimo na PNAD do que nas contas nacionais. Isso porque há, na PNAD, uma propensão de o entrevistado declarar rendimento igual ao salário mínimo.

Tanto nas contas nacionais como na PNAD, a renda per capita das famílias cuja renda segue o salário mínimo é igual a $w_{t}$. No caso da PNAD, a renda per capita das famílias cuja renda não segue o salario mínimo é igual a $v_{t}$. No caso das contas nacionais, o consumo per capita dessas famílias é $z_{t}>v_{t}$, ou seja, supõe-se que toda a subdeclaração de renda que se observa na PNAD provém dessas famílias, seja por estarem no setor informal, seja por disporem de rendas altas que são subdeclaradas.

Então, para simplificar as derivações e sem perda de generalidade, supomos que a renda média per capita na PNAD, $y_{t}$, seja igual à seguinte média geométrica ponderada das rendas dos dois tipos de família ${ }^{3}$ :

$$
y_{t}=w_{t}^{a} v_{t}^{1-a}
$$

De forma similar, o consumo per capita das famílias nas contas nacionais, $c_{t}$, é igual a uma média geométrica ponderada do consumo (igual à renda) dos dois tipos de família ${ }^{4}$ :

$$
c_{t}=w_{t}^{b} z_{t}^{1-b}
$$

\footnotetext{
${ }^{3}$ Embora usemos o mesmo símbolo, o parâmetro $a$ na equação (1) não é idêntico à proporção $a$ definida dois parágrafos antes. Pode-se verificar que um é uma função monotonicamente crescente do outro.

${ }^{4}$ Aplica-se ao parâmetro $b$ o mesmo tipo de observação feito anteriormente para o parâmetro $a$.
} 
Tomando a razão entre (1) e (2) obtemos:

$$
\frac{y_{t}}{c_{t}}=w_{t}^{a-b} \frac{v_{t}^{1-a}}{z_{t}^{1-b}}=w_{t}^{a-b} v_{t}^{b-a}\left(\frac{v_{t}}{z_{t}}\right)^{1-b}
$$

Admitindo que seja constante o grau de subestimação das rendas da PNAD que não seguem o salário mínimo, em comparação com o mesmo tipo de renda (consumo) nas contas nacionais, podemos escrever

$$
\frac{y_{t}}{c_{t}}=w_{t}^{a-b} v_{t}^{b-a} u^{1-b}
$$

onde

$$
u=\frac{v_{t}}{z_{t}}, \text { constante, com } 0<u<1
$$

\section{Definindo}

$$
d=a-b
$$

a expressão (4) fica

$$
\frac{y_{t}}{c_{t}}=w_{t}^{d} v_{t}^{-d} u^{1-b}
$$

ou

$$
\ln y_{t}-\ln c_{t}=d \ln w_{t}-d \ln v_{t}+(1-b) \ln u
$$

Designando por $x_{t}^{\prime}$ a taxa de variação de uma variável $x_{t}$, de (6) ou de (7) segue-se que

$$
y_{t}^{\prime}-c_{t}^{\prime}=d w_{t}^{\prime}-d v_{t}^{\prime}
$$

Para proceder à estimação econométrica de (8), como não dispomos da variável $v_{t}^{\prime}$ vamos substituí-la por seu valor médio no período de análise ${ }^{5}$, o qual designamos simplesmente por $v^{\prime}$ :

$$
v_{t}^{\prime}=v^{\prime} \text { para todo } t
$$

\footnotetext{
${ }^{5}$ Testamos a hipótese mais refinada de que $v_{t}^{\prime}$ fosse não uma constante, mas seguisse uma trajetória de conversão à média do tipo: $v_{t}^{\prime}-v^{\prime}=h\left(w_{t-1}^{\prime}-v^{\prime}\right)$, com $0<h<1$, mas os resultados estatísticos referentes à variável $w_{t-1}^{\prime}$ foram insatisfatórios.
} 
Embora não conheçamos o valor de $v^{\prime}$, ele pode ser inferido a partir das estimativas dos coeficientes de uma regressão.

Para isso estimamos a seguinte relação derivada de (8)

$$
y_{t}^{\prime}-c_{t}{ }^{\prime}=d w_{t}{ }^{\prime}-d v^{\prime}=d\left(w_{t}{ }^{\prime}-v^{\prime}\right)
$$

Dessa forma, o valor de $v^{\prime}$ (taxa suposta constante de crescimento da renda das famílias cuja renda não segue o salário mínimo) pode ser estimado pela razão entre a estimativa da constante, $d v^{\prime}$, e a estimativa do coeficiente $d$ de $w_{t}^{\prime}$. Também se constata que a taxa de crescimento da renda das famílias na PNAD, $y_{t}^{\prime}$, será maior (menor) do que a taxa de crescimento do consumo das famílias nas contas nacionais, $c_{t}^{\prime}$, na medida em que a taxa de crescimento do salário mínimo, $w_{t}^{\prime}$, seja maior (menor) do que a taxa estimada de crescimento do consumo das famílias cuja renda não segue o salário mínimo, $v^{\prime}$.

\section{RESULTADOS ECONOMÉTRICOS}

Os resultados econométricos completos da estimação da relação (10) para o período 1996-2012 estão na Tabela 1. O Quadro 1 contém uma descrição detalhada das variáveis utilizadas na estimação e o Gráfico 3 mostra a evolução dos índices relativos de $y_{t}, c_{t}$, do salário mínimo real e do PIB per capita (média ponderada do terceiro e do quarto trimestres), todos com base em 1996.

Em resumo, nossa estimativa para a equação (10) é:

$$
y_{t}^{\prime}-c_{t}^{\prime}=0,5906 w_{t}^{\prime}-0,02251
$$

de onde inferimos o valor $v^{\prime}=0,02251 / 0,5906=0,0381$. Ou seja, a taxa de crescimento da renda das famílias cuja renda não segue o salário mínimo, estimada por meio da regressão, é igual a 3,8 por cento ao ano.

Dessa forma, nossa conclusão é que sempre que o salário mínimo crescer em termos reais mais do que 3,8 por cento ao ano, a taxa de crescimento da renda das famílias na PNAD será maior do que a taxa de crescimento do consumo das famílias nas contas nacionais. Por exemplo, de acordo com nossas estimativas o crescimento observado de 8 por cento do salário mínimo real em 2012 teria sido responsável por uma diferença de 2,5 pontos percentuais entre o crescimento da renda das famílias na PNAD e o crescimento do consumo das famílias nas contas nacionais. Nesse ano, a diferença efetivamente observada foi de 5,2 pontos de percentagem - ou seja, duas vezes superior à estimada pelo modelo.

De acordo com a relação (5), o coeficiente $d$ de $w_{t}^{\prime}$ é a diferença entre os pesos $a$ e $b$ das rendas que seguem o salário mínimo na formação da renda média na PNAD e nas contas nacionais. O valor estimado desse coeficiente $(d=0,6)$ parece ser muito 
$t$ indica o ano, variando de 1996 a 2012, pulando os anos de 2000 e 2010, nos quais não foi realizada a PNAD. São utilizadas, portanto, séries de 15 valores, gerando 14 diferenças.

$y_{t}$ é o valor real médio, em cada ano, da renda domiciliar per capita (RDPC) obtida da PNAD. Considera-se o Brasil, exclusiva a área rural da antiga região Norte, que não era coberta pela PNAD até 2003. Os valores nominais foram todos colocados em $\mathrm{R} \$$ de setembro-outubro de 2012, usando para isso a média geométrica do INPC nesses dois meses. A ideia é obter um deflator centrado no começo de outubro, tendo em vista que o mês de referência da PNAD no período analisado foi sempre setembro, e a maioria das pessoas recebe o rendimento no começo do mês seguinte.

$$
y_{t}^{\prime}=\Delta \ln y_{t}=\ln y_{t}-\ln y_{t-1}
$$

Seja $S_{t}$ o valor real do salário mínimo no mês de referência da PNAD. O valor real foi calculado usando o mesmo procedimento aplicado à RDPC. Embora $w_{t}$ deva ser diferente de $S_{t}$, é razoável admitir que a taxa de crescimento dessas duas variáveis sejam iguais, ou seja,

$$
w_{t}^{\prime}=\Delta \ln S_{t}=\ln S_{t}-\ln S_{t-1}
$$

No site do IBGE, em "Sistemas de Contas Nacionais Trimestrais", obteve-se séries de índices de base móvel trimestral para o PIB e para o Consumo das Famílias do 1o trimestre de 1996 ao 4o trimestre de 2012. Nesse período o índice de Consumo das famílias variou de 95,88 a 176,76. O valor de $c_{t}$ foi calculado, em cada ano, como uma média ponderada dos índices de Consumo das famílias no terceiro trimestre (com peso $2 / 3$ ) e no quarto trimestre (com peso 1/3). Foi calculado o mesmo tipo de média ponderada para o PIB.

De maneira análoga ao que foi feito para as demais variáveis, define-se

$$
c_{t}^{\prime}=\Delta \ln c_{t}=\ln c_{t}-\ln c_{t-1}
$$

Para 2001 e 2011, devido à inexistência do valor de $y_{t}$ no ano imediatamente anterior, foi utilizada a seguinte definição de $y_{t}^{\prime}$ :

$$
y_{t}^{\prime}=0,5\left(\ln y_{t}-\ln y_{t-2}\right)
$$

Por uniformidade, o mesmo procedimento foi aplicado no cálculo de $w_{t}^{\prime}$ e $c_{t}^{\prime}$ para 2001 e 2011.

Ao ajustar a regressão, as diferenças referentes a 2001 e a 2011 receberam peso 2, por serem médias de 2 anos. 
Gráfico 3: Evolução do salário mínimo real, da RDPC, do PIB e do consumo das famílias, Brasil, 1996-2012

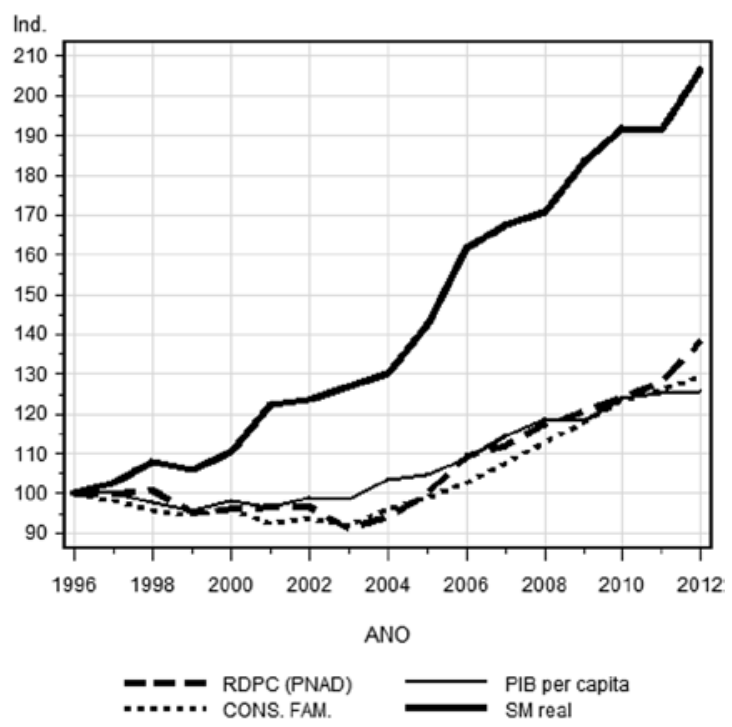

Tabela 1: A equação de regressão de $y_{t}^{\prime}-c_{t}^{\prime}$ contra $w_{t}^{\prime}$ no período 1996-2012

\begin{tabular}{lccc}
\hline \multicolumn{1}{c}{ Estatística } & Estimativa & Teste $t$ & Valor $p(\%)$ \\
\hline Constante & $-0,0225$ & $-2,63$ & 2,2 \\
Coef. de $w_{t}^{\prime}$ & 0,5906 & 3,96 & 0,2 \\
$\mathrm{n}^{\circ}$ de observações $=14, r^{2}=0,57$, teste de Durbin-Watson $=1,92$ & \\
\hline
\end{tabular}

alto, pois, como vimos em seção anterior, a percentagem das pessoas com 10 anos ou mais com renda próxima do salário mínimo varia de 15 a 25 por cento do total nas pesquisas da PNAD. Por mais que estendamos esse entorno, é difícil imaginar que ele pudesse atingir 60 por cento na PNAD e zero por cento nas contas nacionais, conforme necessário para dar consistência ao valor do coeficiente estimado.

Uma possível explicação é que a estimativa desse coeficiente deve estar se apropriando do efeito de outras variáveis positivamente relacionadas com o salário mínimo e que não foram incluídas nas nossas estimativas. Tentamos incluir na equação estimada, como variáveis explicativas adicionais, as variações do crédito bancário às pessoas físicas e também as variações da proporção de trabalhadores no setor informal (definido de distintas formas). Entretanto, essas variáveis não tiveram qualquer poder de explicação das diferenças estimadas entre as variações da renda e do consumo. Também foi estimada uma equação de regressão de $y_{t}^{\prime}-c_{t}^{\prime}$ contra $w_{t}^{\prime}$ e $w_{t-1}^{\prime}$, mas o coeficiente dessa última variável se mostrou claramente não significativo. 


\section{CONCLUSÕES}

Neste texto, testamos a hipótese de que as variações do salário mínimo explicariam as diferenças observadas entre as taxas de variação da renda das famílias na PNAD e as do consumo das famílias nas contas nacionais, utilizando dados anuais para o período entre 1996 e 2012. Demonstramos que essa hipótese segue-se imediatamente à suposição de que a subestimativa de renda na PNAD é menor entre as famílias que recebem o salário mínimo.

Concluímos que o comportamento do salário mínimo teve um papel importante na explicação das diferenças entre as variações da renda na PNAD e as do consumo das famílias nas contas nacionais. Especificamente, segundo nossas estimativas, sempre que o salário mínimo cresce mais do 3,8 por cento ao ano em termos reais, há uma diferença positiva entre o crescimento da renda da PNAD e o crescimento do consumo das famílias nas contas nacionais.

O coeficiente de regressão que estimamos para o salário mínimo parece exagerado em face da concentração das rendas declaradas em torno dele, que observamos na PNAD. Essa superestimação pode estar ligada às simplificações que tivemos que adotar, supondo constantes valores que efetivamente variam ao longo do tempo, ou a não termos conseguido identificar outras variáveis que teriam um efeito explicativo adicional para as diferenças entre a PNAD e as contas nacionais. O salário mínimo pode assim estar captando também o efeito dessas variáveis omitidas.

Mesmo com essa aparente superestimativa do efeito do salário mínimo, constatamos que apenas metade da diferença entre o crescimento da renda da PNAD e o do consumo das famílias em 2012 pode ser imputada ao forte crescimento do salário mínimo nesse ano.

A conclusão é que, embora tenhamos conseguido identificar parte da explicação da diferença entre as contas nacionais e a PNAD, ainda restam enigmas adicionais a serem decifrados por pesquisas posteriores.

\section{REFERÊNCIAS BIBLIOGRÁFICAS}

BACHA, Edmar (2013). “O pibinho e a pnadona”, O Globo, 8/10/2013, disponível em: http://www. iepecdg.com.br/uploads/texto/globo-6c64zqe6ztt1cd0nibc5_original\%20maranh\%C3\%A3o.pdf

BAUMGARTEN DE BOLLE, Monica e SIMÔES, Pedro H. de Castro (2013). "O espantalho, o homem de lata e o leão: a PNAD, o PIB e o consumo em busca do Mágico de Oz”. Carta Econômica Galanto 163/13, set. 2013, p. 8-15. Disponível em: http://www.iepecdg.com.br/uploads/artigos/ Carta\%20Econ\%C3\%B4mica\%20Galanto\%20-\%200913.pdf

HOFFMANN, Rodolfo (2008). Polarização da distribuição de renda no Brasil. Econômica 10(2):169186, dez. 2008.

IPEA (2013). Duas décadas de desigualdade e pobreza no Brasil medidas pela PNAD/IBGE. Comunicados do IPEA n. 159, 2013.

SOARES, Sergei S.D. (2004). O impacto distributivo do salário mínimo: a distribuição individual dos rendimentos do trabalho. Economia Aplicada 8(1): 47-76, jan.-mar. 2004. 